\title{
Outcome after reconstruction of discontinuous pulmonary arteries
}

\author{
Christof Stamm, MDa \\ Ingeborg Friehs, MD \\ David Zurakowski, $\mathrm{PhD}^{\mathrm{b}}$ \\ Albertus M. Scheule, MD* \\ Adrian M. Moran, MD** \\ James E. Lock, MD \\ John E. Mayer, Jr, MDa \\ Pedro J. del Nido, $\mathrm{MD}^{\mathrm{a}}$ \\ Richard A. Jonas, MDa
}

Objective: This study was undertaken to determine outcomes of and optimal treatment strategies for reconstruction of congenital or acquired discontinuity of branch pulmonary arteries.

Methods: Between 1985 and 2000 pulmonary artery continuity was established in 102 patients with discontinuous central pulmonary arteries and normal peripheral arborization. Data were obtained retrospectively.

Results: Techniques to connect both pulmonary arteries included direct pulmonary artery-pulmonary artery anastomosis $(n=33)$, tube graft interposition $(n=47)$, or pulmonary arterial implantation in right ventricular-pulmonary arterial conduits (n $=22)$. Among patients with biventricular repair $(\mathrm{n}=66)$, survival was $85 \% \pm 8 \%$ at 5 years, and freedom from surgical or interventional pulmonary arterioplasty was $31 \% \pm 11 \%$. At most recent follow-up, mean branch pulmonary arterial $z$ scores were $-0.5 \pm 1.6$ (right pulmonary artery) and $-1.4 \pm 1.3$ (left pulmonary artery). Mean right to left ventricular pressure ratio was $0.61 \pm 0.26$, and this value was more than 0.75 in 13 of 58 cases. Fifteen of 51 had a lung perfusion mismatch of more than 75:25, and in 9 of 58 one branch pulmonary artery was occluded. Twenty-two patients who underwent primary establishment of antegrade pulmonary artery flow without previous shunt procedures had comparable survival and reintervention rates, with a tendency toward higher pulmonary arterial $z$ scores and lower right to left ventricular pressure ratios. Among patients with single-ventricle repair $(\mathrm{n}=33), 5$-year survival was $93 \% \pm 8 \%$ and freedom from pulmonary arterioplasty was $39 \% \pm 9 \%$. Ten of 19 patients had a lung perfusion mismatch, and one branch pulmonary artery was occluded in 4 of 31 . Overall, a direct pulmonary artery anastomosis was associated with better survival $(P=.006)$. The presence of aortopulmonary collaterals was a risk factor for pulmonary artery occlusion $(P=.03)$.

Conclusion: Good survival can be achieved for patients with pulmonary artery discontinuity, but this requires frequent reinterventions. Direct pulmonary arterypulmonary artery anastomoses and control of all collateral vessels may further improve outcome.

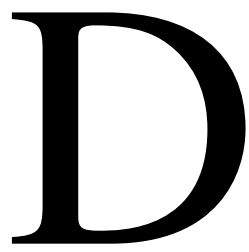

iscontinuity of left and right pulmonary arteries (PAs) is found as a complicating feature of several congenital heart malformations. The main PA or PA bifurcation can be absent in patients with pulmonary atresia, as has been described in up to $16 \%$ of the patients with Fallot's tetralogy and pulmonary atresia. ${ }^{1,2}$ Less frequently, in patients with patent but stenotic right ventricular outflow tract, 
one branch PA can be in continuity with the main PA and the right ventricle (RV) while at the same time the most proximal portion of the contralateral PA is absent or atretic. In the setting of pulmonary atresia or stenosis and PA discontinuity, one or both branch PAs are usually fed by a patent ductus arteriosus or single ductlike collateral vessel, but also multiple aortopulmonary collateral arteries (APCAs) have been described to supply a PA that is not in continuity with a ventricular outflow tract. ${ }^{3}$ In a number of patients with truncus arteriosus (Collet and Edwards type 3b/c, Van Praagh type A3) only one PA arises from the common arterial trunk, with the contralateral PA originating from other structures. ${ }^{4,5}$ Another rare malformation is the origin of either left or right PA from the ascending aorta (hemitruncus) with the contralateral PA in continuity with the main PA and RV. ${ }^{6-8}$ PA discontinuity can also be acquired from construction of end-to-end aortopulmonary shunts or cavopulmonary shunts, such as a unilateral cavopulmonary end-to-side or end-to-end anastomosis (classic Glenn shunt). Furthermore, the central portion of anatomically continuous intrapericardial PAs can be severely distorted and occluded after implantation of a central aortopulmonary shunt or Blalock-Taussig shunt or can spontaneously occlude after birth as the result of abnormal extension of ductal tissue. ${ }^{9}$

Significant problems such as PA hypoplasia and residual or recurrent PA stenoses often develop after reconstruction of PA continuity, resulting in pulmonary perfusion deficits and right ventricular hypertension. There is little information regarding the fate of true PAs after repair of central discontinuity, and the optimal surgical strategy for reconstruction of PA continuity in the context of either univentricular or biventricular repair of the underlying malformation remains to be defined. We therefore reviewed our institutional experience with patients who underwent surgical reconstruction of discontinuous PAs and sought to determine long-term results as well as surgical and anatomic risk factors for recurrent PA stenoses.

\section{Methods \\ Patient Selection}

The goal of the patient selection process was to collect a cohort of patients with discontinuous central PAs in the setting of normal or near-normal distal arborization in whom functional continuity of the PAs was constructed surgically. The principal inclusion criteria were (1) functional discontinuity of left and right PAs; (2) a PA diameter that allowed an end-to-end or end-to-side anastomosis with a graft or native vessel, usually at least $3 \mathrm{~mm}$ in neonates or infants; (3) absence of a multitude of APCAs directly and exclusively supplying a substantial number of lung segments.

All patients with discontinuity of the central (intrapericardial or prebranching extrapericardial) PAs who underwent operations between 1985 and 2000 at Children's Hospital, Boston, were selected from the cardiac surgery and cardiology databases.
Preoperative angiograms and operative reports were then reviewed. Patients with congenitally discontinuous PAs who underwent establishment of PA continuity were included in the study. Also included were patients who developed discontinuity of central PAs after implantation of aortopulmonary shunts or Glenn shunts and who subsequently underwent reconstruction of PA continuity. Patients were excluded if one or more lung segments were directly and exclusively supplied by APCAs, necessitating complex unifocalization procedures to incorporate the APCAs into the pulmonary arterial blood supply. Patients in whom one or more APCAs were part of a dual blood supply to one or more lung segments together with true PA branches were not excluded. Those collateral vessels could be occluded by surgical ligation or intravascular coil placement without interruption of blood flow to the respective segments and did not have to be unifocalized.

\section{Data Acquisition}

The protocol was approved by the institutional review board at Children's Hospital. Hospital charts and charts of cardiologists following up patients were reviewed, as were the computerized databases of Children's Hospital and the Departments of Cardiac Surgery and Cardiology. Information on the underlying cardiac diagnosis, diagnostic catheterizations and echocardiograms, all surgical and interventional procedures, and all available follow-up information were collected retrospectively. Because more than one surgical procedure had been performed in most cases, the operation that established functional continuity of the central PAs was considered the baseline for each patient, and all calculations regarding postoperative follow-up were based on that date. Follow-up information was obtained during 2000 and was complete for 82 of the surviving patients $(98 \%)$, with a mean followup time of $5.4 \pm 3.7$ years.

\section{Hemodynamic and Morphometric Measurements}

Data were obtained during routine clinical follow-up and were collected retrospectively. Preoperative cardiac catheterization had been performed in 94 patients; in the remaining 8 patients only echocardiography had been performed before the operation. Cardiac catheterization during follow-up was performed in 89 cases (mean interval to last catheterization $3.3 \pm 2.9$ years). In 2 additional cases right ventricular pressure at follow-up was derived from the peak velocity of the tricuspid regurgitation jet as measured by Doppler echocardiography. For patients who had undergone a biventricular repair, the systolic right ventricular to systemic pressure ratio was calculated to assess right ventricular pressure load. In cases in which the left ventricle was not entered at catheterization, the right ventricular to systemic pressure ratio was calculated on the basis of the systolic aortic blood pressure. The pressure gradient between the most central part of the reconstructed PA confluence or the most distal part of an RV-PA conduit and the distal PAs was recorded. For patients who underwent definitive univentricular palliation with a bidirectional Glenn shunt or Fontan operation, the pressure in the superior vena cava (SVC) was recorded and pressure gradients between the SVC or Fontan conduit and central PAs were noted. PA diameter was measured on anteroposterior angiograms, and $z$ scores of PA size for a given body surface area were calculated from the diameter mea- 

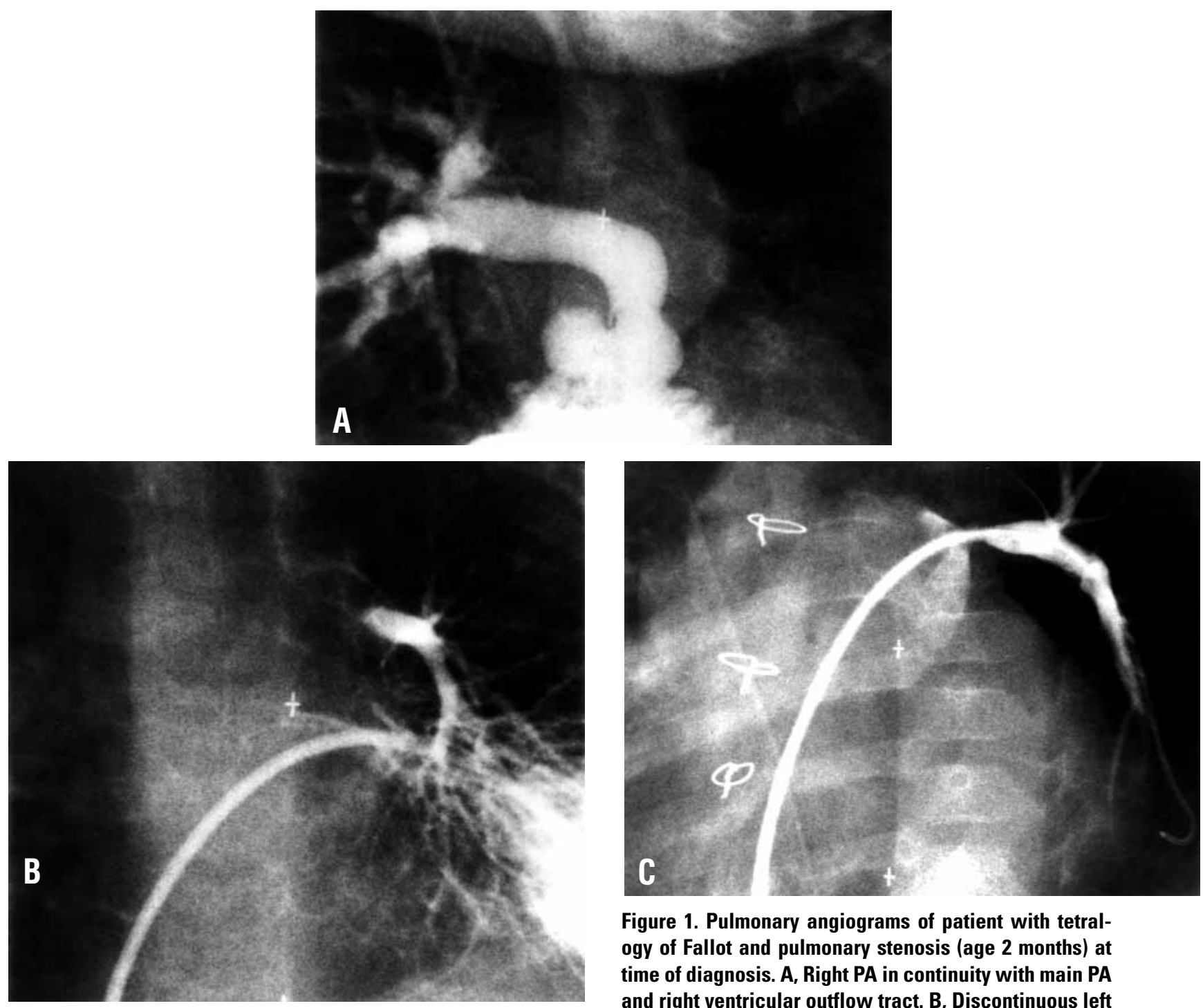

Figure 1. Pulmonary angiograms of patient with tetralogy of Fallot and pulmonary stenosis (age 2 months) at time of diagnosis. A, Right PA in continuity with main PA and right ventricular outflow tract. $B$, Discontinuous left PA originating from undersurface of distal aortic arch by way of patent ductus arteriosus. C, Pulmonary angiogram 5 months after primary repair (VSD closure, right ventricular outflow tract patch, and direct anastomosis of left PA to main PA). There is significant stenosis of left PA at site of anastomosis. Balloon dilatation and stent placement were subsequently performed and led to angiographic and hemodynamic improvement.

sured just proximal to the first branching point. Two-dimensional echocardiography was used to measure the diameter of the central PAs at most recent follow-up in 13 patients. Calculations of $z$ scores were based on the normal ranges recorded in an institutional database representative of the local patient demographic characteristics.

\section{Statistical Analysis}

Estimated rates of survival and freedom from reintervention with 95\% confidence intervals (CIs) were determined by the KaplanMeier product-limit method, and curves were compared by the log-rank test. Continuous and categorical variables tested in the univariable and multivariable analyses are listed in the Appendix. The Cox proportional-hazards regression model was used to estab- lish the variables independently associated with death and reintervention with risk measured by the adjusted hazard ratio. Multiple logistic regression analysis was performed to determine the risk factors independently predictive of PA occlusion, with the likelihood ratio $\chi^{2}$ test used to assess significance. For both the Cox model and logistic regression a backward stepwise selection procedure was used to build the final multivariate models. Hemodynamic and morphometric variables were evaluated by the Kolmogorov-Smirnov test to ascertain whether the measurements followed a normal distribution. For variables with a non-Gaussian distribution, the Mann-Whitney $U$ test was used to compare groups and the Wilcoxon signed rank test was used for comparisons between preoperative and follow-up data, and those data are presented as median and range. All variables with a normal distri- 


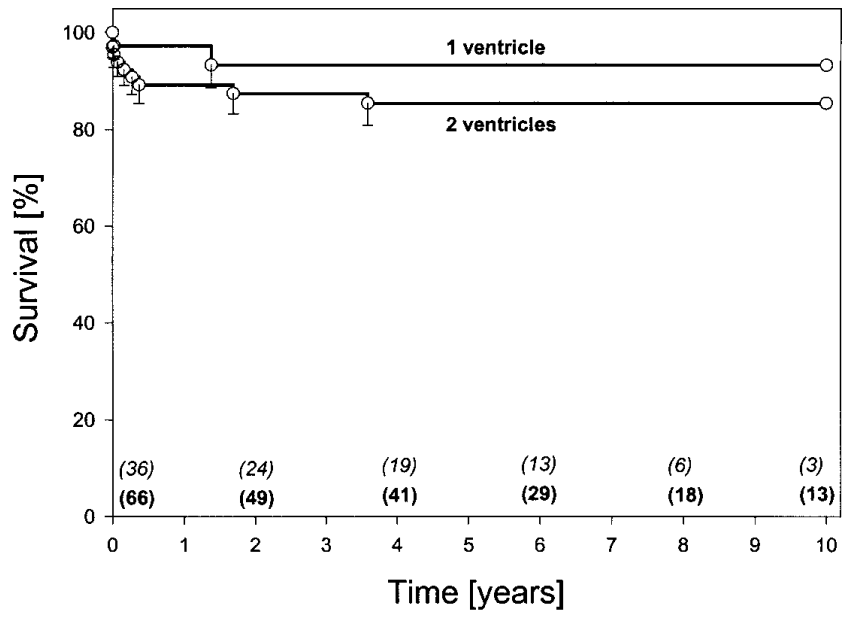

Figure 2. Kaplan-Meier estimated survivals after reconstruction of discontinuous PAs in patients with functionally univentricular hearts undergoing single ventricle palliation with ultimate Fontan procedure (1 ventricle) and patients undergoing biventricular repair of underlying heart defect (2 ventricles). Error bars indicate lower $\mathbf{9 5} \% \mathrm{CI}$. Survival was not statistically significantly different between groups ( $P=.6$ by log-rank test).

bution are expressed as mean \pm SD and were analyzed with the appropriate paired or unpaired $t$ test. Analysis of the data was performed with the SPSS software package (version 10.0; SPSS Inc, Chicago, Ill).

\section{Results}

\section{Patient Demographic Characteristics}

A total of 102 patients were included in the study. Fifty-three patients $(52 \%)$ were male and $49(48 \%)$ were female. The age at the time of establishment of PA continuity ranged between 5 days and 35 years (median 2.6 years). The weight at operation ranged between $2 \mathrm{~kg}$ and $75 \mathrm{~kg}$ (median 10.2 $\mathrm{kg}$ ). The underlying anatomic diagnoses included tetralogy of Fallot with pulmonary atresia $(n=39)$, tetralogy of Fallot with patent right ventricular outflow tract $(n=15)$, other forms of pulmonary atresia $(n=21)$, tricuspid atresia $(n=$ $12)$, atypical truncus arteriosus $(n=5)$, and others $(n=10)$. PA discontinuity was congenital in 74 cases $(73 \%)$ and acquired after construction of systemic-to-PA shunts in 28 cases $(27 \%)$. Among patients who underwent single-ventricle repair, PA discontinuity was often a shunt complication (acquired in 20/36 [55\%]), whereas for most of the patients who underwent biventricular repair, PA discontinuity was a component of the child's anomaly (acquired in 8/66 [12\%]).

\section{Preoperative Pulmonary Circulation}

In 81 patients $(80 \%)$ the main PA was absent or atretic and therefore both left and right PAs were supplied by either a shunt, a persistent ductus arteriosus (PDA), or one or more APCAs. In the remaining 21 patients $(20 \%)$ there was a

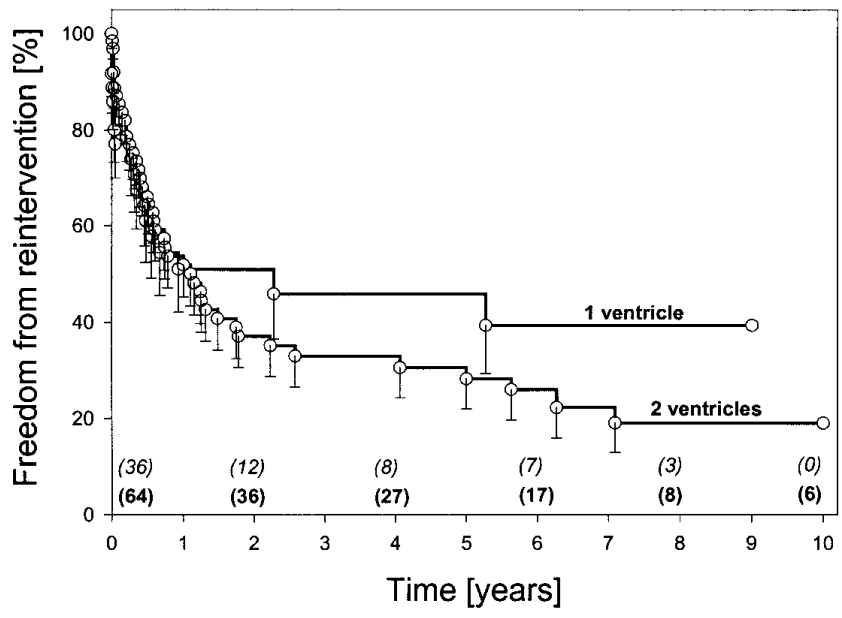

Figure 3. Kaplan-Meier estimated freedoms from pulmonary angioplasty (including both surgical patch angioplasty and interventional balloon angioplasty) after reconstruction of discontinuous PAs in patients with functionally univentricular hearts undergoing single ventricle palliation with ultimate Fontan procedure (1 ventricle) and patients undergoing biventricular repair of underlying heart defect (2 ventricles). Error bars indicate lower $95 \% \mathrm{CI}$. Freedom from angioplasty was not statistically significantly different between groups ( $P=.5$ by log-rank test).

main PA connecting one branch PA with a patent ventricular outflow tract, with the contralateral PA being discontinuous and supplied by a shunt, persistent ductus arteriosus, or collateral vessel (Figure 1). Additional APCAs were present in 31 patients $(30 \%)$ and had been occluded by interventional coil placement in 20 cases. Before the establishment of PA continuity a total of 78 patients $(76 \%)$ had undergone a palliative shunt procedure. The diameters of left and right PAs varied between $3 \mathrm{~mm}$ and $18 \mathrm{~mm}$. Right PA $z$ scores ranged between -5.15 and 5.2 and were lower than -2 in 18 cases. Left PA $z$ scores ranged between -5.4 and 3.9 and were lower than -2 in 15 cases. Systolic right and left pulmonary arterial pressures ranged between 8 and $115 \mathrm{~mm} \mathrm{Hg}$ (median $23 \mathrm{~mm} \mathrm{Hg}$ ), and 35 patients had a mean pressure in the right or left PA greater than $30 \mathrm{~mm}$ $\mathrm{Hg}$. In 53 cases preoperative lung perfusion scans had been performed, and 18 of those (34\%) indicated a perfusion mismatch exceeding 75:25. Preoperative balloon dilatation of PA stenoses had been performed in 20 cases, including intravascular stent placement in 3 .

\section{Biventricular Repair}

In a total of 66 cases the intracardiac anatomy allowed a biventricular repair. Forty-four of these patients $(67 \%)$ had undergone previous implantation of systemic-to-PA shunts to increase blood flow in the discontinuous PA, in contrast to 22 patients (33\%) in whom both PA continuity and antegrade pulmonary blood flow through a reconstructed RV 
TABLE 1. Mortality in patients undergoing repair of discontinuous pulmonary arteries

\begin{tabular}{|c|c|c|c|c|c|c|c|}
\hline & No. & Diagnosis & Age & $\begin{array}{c}\text { Year of } \\
\text { operation }\end{array}$ & Operation & Complications & $\begin{array}{l}\text { Time to } \\
\text { death }\end{array}$ \\
\hline \multirow[t]{9}{*}{ Biventricular } & 1 & $\begin{array}{l}\text { Fallot, } \\
\text { discontinuous LP }\end{array}$ & $16 d$ & 1990 & $\begin{array}{l}\text { Fallot repair with transannular } \\
\text { RVOT patch, LPA-MPA direct } \\
\text { anastomosis }\end{array}$ & RV failure & $8 d$ \\
\hline & 2 & $\begin{array}{l}\text { Truncus arteriosus, } \\
\text { discontinuous RPA }\end{array}$ & $2 y$ & 1993 & $\begin{array}{l}\text { RV-LPA homograft with RPA } \\
\text { implantation, fenestrated VSD patch }\end{array}$ & $\begin{array}{l}\text { LV failure after surgical } \\
\text { VSD closure }\end{array}$ & $5 \mathrm{mo}$ \\
\hline & 3 & $\begin{array}{l}\text { DORV, TGA, pulmonary } \\
\text { atresia, PA discontinuity }\end{array}$ & $2.2 \mathrm{y}$ & 1990 & $\begin{array}{l}\text { RV-PA homograft, RPA + LPA } \\
\text { implantation, VSD closure }\end{array}$ & Viral pneumonia, ARDS & $26 \mathrm{~d}$ \\
\hline & 4 & $\begin{array}{l}\text { Fallot + pulmonary atresia, } \\
\text { PA discontinuity }\end{array}$ & $4 d$ & 1998 & $\begin{array}{l}\text { RV-LPA homograft, RPA } \\
\text { implantation, VSD closure }\end{array}$ & Cardiac arrest at home & $2 \mathrm{mo}$ \\
\hline & 5 & $\begin{array}{l}\text { Fallot, hypoplastic LPA, } \\
\text { RPA discontinuity }\end{array}$ & $1.2 \mathrm{mo}$ & 1989 & $\begin{array}{l}\text { RV-RPA homograft, VSD closure, } \\
\text { LPA not reanastomosed }\end{array}$ & Hypoxia, sepsis & $1.2 \mathrm{mo}$ \\
\hline & 6 & $\begin{array}{l}\text { Fallot + pulmonary atresia, } \\
\text { LPA from ascending aorta }\end{array}$ & $3.4 \mathrm{y}$ & 1995 & $\begin{array}{l}\text { LPA-RPA direct anastomosis, } \\
\text { RV-PA homograft, fenestrated } \\
\text { VSD patch }\end{array}$ & Biventricular failure & $0 \mathrm{~d}$ \\
\hline & 7 & $\begin{array}{l}\text { Fallot + pulmonary atresia, } \\
\text { PA discontinuity }\end{array}$ & $4.4 \mathrm{y}$ & 1989 & $\begin{array}{l}\text { RV-PA conduit, RPA + LPA } \\
\text { implantation, VSD left open }\end{array}$ & $\begin{array}{l}\text { Biventricular failure after } \\
\text { RV-PA conduit exchange }\end{array}$ & $1.7 y$ \\
\hline & 8 & $\begin{array}{l}\text { Fallot + pulmonary atresia, } \\
\text { PA discontinuity }\end{array}$ & $11 \mathrm{mo}$ & 1991 & $\begin{array}{l}\text { RV-RPA homograft, tube graft } \\
\text { to LPA, VSD closure }\end{array}$ & LPA occlusion, hypoxia & $3.6 y$ \\
\hline & 9 & $\begin{array}{l}\text { Fallot + pulmonary atresia, } \\
\text { PA discontinuity }\end{array}$ & $3.2 \mathrm{y}$ & 1989 & $\begin{array}{l}\text { Left modified Blalock-Taussig } \\
\text { shunt, RPA-LPA anastomosis }\end{array}$ & $\begin{array}{l}\text { Postoperative cardiac } \\
\text { arrest }\end{array}$ & $0 d$ \\
\hline \multicolumn{8}{|l|}{ Univentricular } \\
\hline & 10 & $\begin{array}{l}\text { Tricuspid atresia + } \\
\text { pulmonary atresia, } \\
\text { acquired PA discontinuity }\end{array}$ & $3 \mathrm{mo}$ & 1994 & $\begin{array}{l}\text { RPA-LPA tube graft, PDA, } \\
\text { central shunt, followed } \\
\text { by bidirectional Glenn shunt }\end{array}$ & Hypoxia, cardiac arrest & $1.4 \mathrm{y}$ \\
\hline & 11 & $\begin{array}{l}\text { Tricuspid atresia + } \\
\text { pulmonary atresia, } \\
\text { acquired PA discontinuity }\end{array}$ & $16 y$ & 1998 & $\begin{array}{l}\text { Lateral tunnel conversion for } \\
\text { failing atriopulmonary Fontan, } \\
\text { tube graft SVC-LPA }\end{array}$ & Postoperative LV failure & $7 d$ \\
\hline
\end{tabular}

$L P A$, Left pulmonary artery; $R V O T$, right ventricular outflow tract; $M P A$, main pulmonary artery; $R V$, right ventricular; $R P A$, right pulmonary artery; $L V$, left ventricular; VSD, ventricular septal defect; $D O R V$, double-outlet right ventricle; TGA, transposition of the great arteries; $P A$, pulmonary artery; $A R D S$, acute respiratory distress syndrome; PDA, patent ductus arteriosus; SVC, superior vena cava.

outflow tract were achieved without previous shunt implantation. PA continuity was achieved by direct end-to-end anastomosis between both PAs or direct end-to-side implantation of the discontinuous PA into the main PA in 17 patients $(26 \%)$. A polytetrafluoroethylene or homograft tube graft was used to connect both branch PAs in 28 patients (42\%), and both PAs were directly implanted into an RV-to-PA homograft in 21 patients. In 53 cases PA continuity was established together with final repair of the intracardiac malformation including closure of a ventricular septal defect (VSD), in 9 cases PA continuity was achieved together with RV-PA continuity but the VSD was left open, and in 4 cases PA continuity was established and a central shunt or modified Blalock-Taussig shunt was implanted for pulmonary blood supply. Fifteen patients had total onestage repair including closure of the VSD without previous shunt operations, and in 3 patients a fenestrated VSD patch was used. A total of 57 (86\%) patients had their biventricular repair completed at last follow-up.

Survival. There were 6 early deaths $(9 \%)$ and 3 late deaths (5\%) after establishment of PA continuity among patients undergoing biventricular repair (Table 1). KaplanMeier-estimated survivals were $90 \% \pm 7 \%$ at 1 year and $85 \% \pm 8 \%$ at 5 years (Figure 2).

Reinterventions. A total of 35 surgical procedures were performed in 28 patients $(42 \%)$ after establishment of PA continuity. Patch enlargement of central PA stenoses was performed in 10 cases (15\%), and 44 patients $(67 \%)$ underwent as many as five interventional balloon dilatations of PA stenoses, which in 30 cases included placement of intravascular stents. When both surgical and catheter-based procedures are combined, the freedoms from pulmonary angioplasty after construction of PA continuity were $54 \% \pm$ $10 \%$ at 1 year and $31 \% \pm 11 \%$ at 5 years (Figure 3 ). Other 


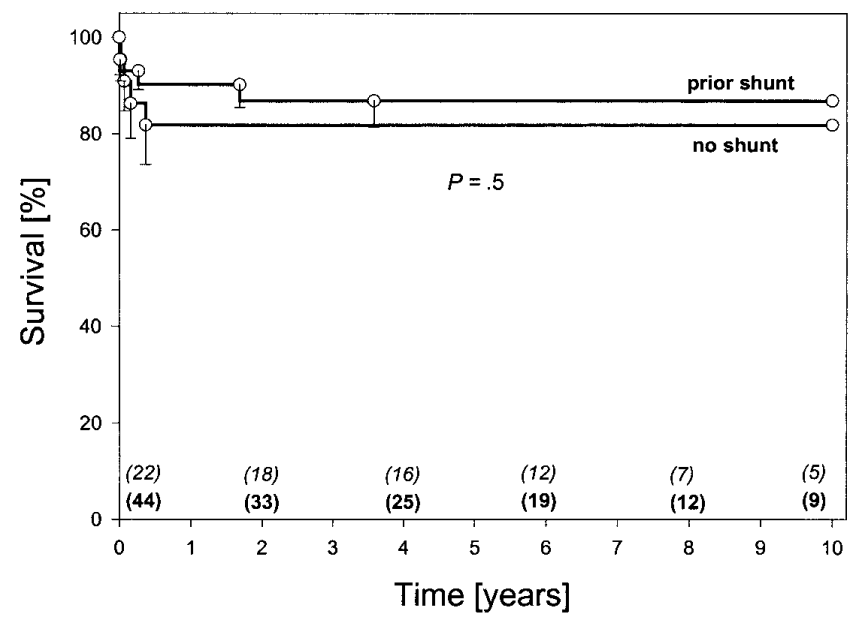

Figure 4. Kaplan-Meier estimated survivals after reconstruction of discontinuous PAs in patients undergoing biventricular repair either after implantation of systemic-to-PA shunt before reconstruction of PA continuity (prior shunt) or with establishment of antegrade PA flow and reconstruction of PA continuity without previous shunt procedures (no shunt). Error bars indicate lower $95 \% \mathrm{Cl}$.

reoperations included exchange of a stenotic RV-PA homograft conduit in 16 patients and closure of a VSD in 6 patients. An RV-PA conduit was implanted to complete the repair in 3 patients who had previous shunt palliation. Overall Kaplan-Meier-estimated freedoms from reoperation were $81 \% \pm 9 \%$ at 1 year and $53 \% \pm 12 \%$ at 5 years. In 13 patients $(20 \%)$ APCAs were occluded by intravascular coil placement during follow-up, and 4 patients underwent catheter-based device closure of a VSD patch fenestration.

Pulmonary circulation. At the time of the most recent cardiac catheterization, mean systolic right ventricular pressure was $59.6 \pm 24 \mathrm{~mm} \mathrm{Hg}$, ranging between 30 and 110 $\mathrm{mm} \mathrm{Hg}$ among patients with completed biventricular repair. The right ventricular to systemic pressure ratio ranged between 0.18 and 1.2 , with a mean of $0.61 \pm 0.26$. Thirteen patients $(20 \%)$ had right ventricular to systemic pressure ratios greater than 0.75; all of these underwent at least one balloon dilatation to relieve residual PA stenoses, 4 underwent coil occlusion of large APCAs as the presumed reason for the PA hypertension, and 3 died during follow-up. The median calculated pulmonary vascular resistance was 3.8 Wood units (range 0.9-30 Wood units). Eleven patients (17\%) had a residual net right-to-left shunt. The mean peripheral oxygen saturation in room air at last follow-up was $94 \% \pm 5 \%$. Median systolic pulmonary arterial pressures were $33 \mathrm{~mm} \mathrm{Hg}(15-92 \mathrm{~mm} \mathrm{Hg})$ in the right PA and $36 \mathrm{~mm} \mathrm{Hg}(16-110 \mathrm{~mm} \mathrm{Hg})$ in the left PA. Twenty-four patients $(36 \%)$ had PA systolic pressures greater than 45 $\mathrm{mm} \mathrm{Hg}$. Twenty-eight patients (42\%) had peak end-systolic

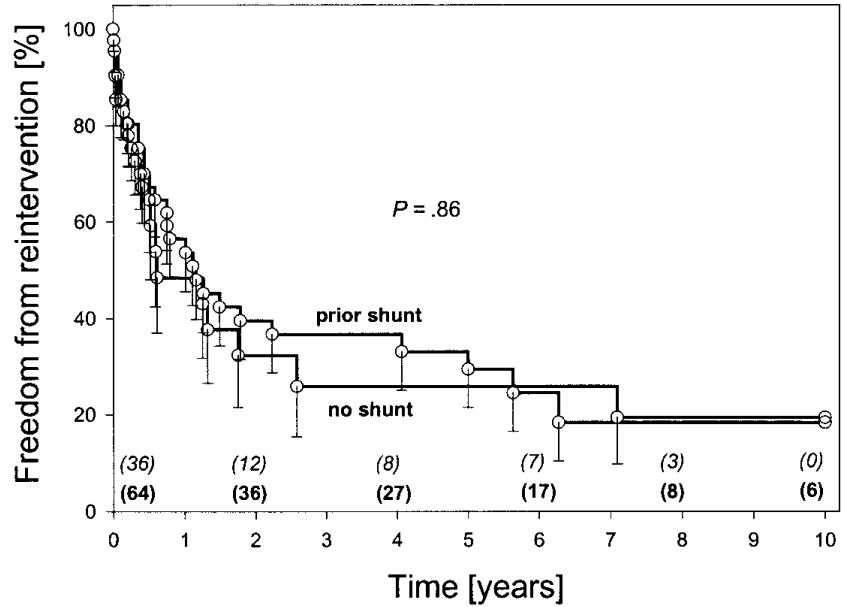

Figure 5. Kaplan-Meier estimated freedom from pulmonary angioplasty (including both surgical patch angioplasty and interventional balloon angioplasty) after reconstruction of discontinuous PAs in patients undergoing a biventricular repair either after implantation of systemic-to-PA shunt before reconstruction of PA continuity (prior shunt) or with establishment of antegrade PA flow and reconstruction of PA continuity without previous shunt procedures (no shunt). Error bars indicate lower $95 \% \mathrm{CI}$.

gradients greater than $25 \mathrm{~mm} \mathrm{Hg}$ between the distal branch PA and the main PA or distal RV-PA conduit.

Right PA $z$ scores at last follow-up ranged from -4.7 to 2.2 and were lower than -2 in 6 cases (9\%). Left PA $z$ scores ranged between -4.4 and 1.3 and were lower than -2 in 14 cases $(21 \%)$. In 51 cases lung perfusion scans had been performed during recent follow-up, and 15 of those showed a lung perfusion mismatch exceeding 75:25. In 9 patients one branch PA was found occluded during follow-up and was successfully recanalized with streptokinase thrombolysis, balloon dilatation, or both with stent placement in 3 patients, or surgically in 3 patients.

Previous shunt versus direct repair. Kaplan-Meierestimated survival did not differ between patients who underwent primary establishment of antegrade PA flow and patients who had previous shunt operations $(82 \% \pm 14 \%$ for shunt vs $86 \% \pm 9 \%$ for no shunt at 5 years, $P=.5$, log-rank test, Figure 4), although the age at operation was significantly younger (median 0.25 years, range $0.2-1.1$ years for no shunt vs median 5.1 years, range 1.5-11.2 years for shunt, $\mathrm{P}<.001)$. Both groups underwent similar numbers of reinterventions or reoperations for PA stenosis (freedom from PA plasty $48 \% \pm 14 \%$ at 1 year and $26 \% \pm 19 \%$ at 5 years for no shunt vs $54 \% \pm 14 \%$ at 1 year and $29 \% \pm 16 \%$ at 5 years for shunt, $P=.9$, Figure 5). Of the 22 patients with primary establishment of antegrade PA flow, 21 (95\%) had the biventricular repair completed, and in 1 case the VSD could not be closed because of severe recurrent bilateral PA stenoses despite four interventional pulmonary 

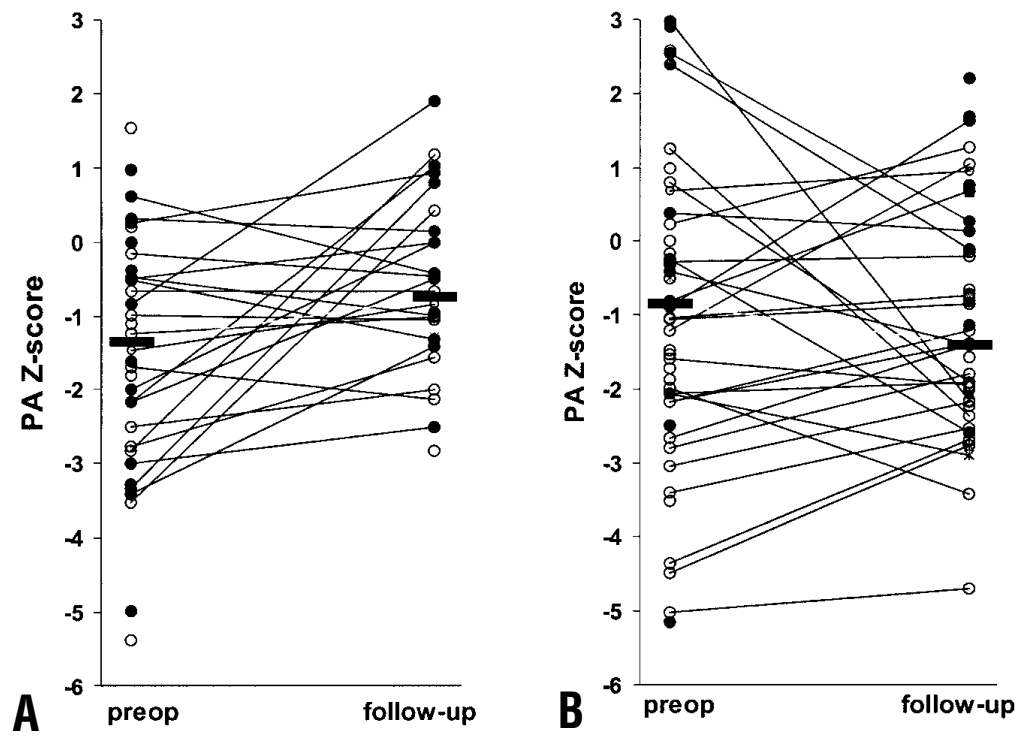

Figure 6. Branch PA z scores in patients who had primary establishment of antegrade PA flow without previous shunt operations (A) and patients who had shunt operations to increase PA blood flow before construction of PA continuity (B). Filled circles, Right PA z scores; open circles, left PA z scores. Respective medians are indicated by horizontal bars.

angioplasties during follow-up. Of the 44 patients who had undergone initial shunt palliation, 37 (84\%) had the repair completed at most recent follow-up. Two patients died before completion of the repair, in 3 patients the VSD could not be closed to date because of residual severe bilateral PA stenoses, and 2 patients are awaiting final repair. A direct PA-to-PA anastomosis was possible in $9(41 \%)$ of the 22 patients with primary establishment of antegrade PA flow but in only $8(18 \%)$ of the 44 patients with initial shunt palliation $\left(P=.05, \chi^{2}\right.$ test). Patients who underwent primary establishment of antegrade PA flow tended to have higher left PA $z$ scores at follow-up than did patients who had previous shunt operations (median -1.02 , range -4.4 to 1.8 for no shunt vs median -1.97 , range -3.4 to 1.3 for shunt, $P=$ .23 ), and this trend was also observed for right PA $z$ scores (median $=-0.21$, range -2.5 to 1.9 for no shunt vs median -0.72 , range -4.7 to 2.2 for shunt, $P=.3$ ). More importantly, follow-up right and left PA $z$ scores were significantly higher than preoperative $z$ scores in patients without previous shunt implantation $(P=.01$ and $\mathrm{P}=.015$, respectively, paired $t$ test, Figure $6, A$ ), but were not different in patients with previous shunt implantation for right PA and left PA $z$ scores $(P=.9$ and $P=.6$, respectively, Figure $6, B)$. Patients who underwent primary establishment of antegrade PA flow tended to have lower pulmonary vascular resistance at follow-up than did patients who had previous shunt operations (median 2.9, range 1.5-30 for no shunt vs median 4.2, range 0.9-19 for shunt, $P=.08$ ). Patients who underwent primary establishment of antegrade PA flow also tended to have lower right ventricular to systemic pressure ratios at follow-up than did patients who had previous shunt operations (median 0.49, range 0.29-1.2 for shunt vs median 0.62, range 0.18-1.0 for no shunt, $P=.06$ ). Pressure gradients between the main PA or RV-PA conduit and the proximal branch PAs were not different between patients who underwent primary establishment of antegrade PA flow and those who had previous shunt operations (median 21 $\mathrm{mm} \mathrm{Hg}$, range 0-70 $\mathrm{mm} \mathrm{Hg}$ for shunt vs median $18 \mathrm{~mm} \mathrm{Hg}$, range $0-100 \mathrm{~mm} \mathrm{Hg}$ for no shunt).

\section{Univentricular Repair}

In 36 patients the intracardiac anatomy did not allow biventricular repair. Thirty of these patients $(83 \%)$ had undergone previous implantation of systemic-to-PA shunts to increase blood flow in the discontinuous PAs, with 9 of these patients also undergoing a classic Glenn shunt. PA continuity was achieved by direct end-to-end anastomosis between both PAs or direct end-to-side implantation of a unilateral discontinuous PA into the main PA in 16 cases (44\%). A polytetrafluoroethylene or homograft tube graft was used to connect both branch PAs in 19 cases (53\%), and both PAs were directly implanted into an extracardiac inferior vena cava-PA conduit in one patient (3\%). In 13 cases PA continuity was achieved together with implantation of a central shunt or modified Blalock-Taussig shunt for pulmonary blood supply, in 16 cases in association with construction of a bidirectional Glenn shunt, and in 7 patients establishment of PA continuity was part of the final Fontan repair. A total 
TABLE 2. Predictors of death, reintervention, and PA occlusion after establishment of PA continuity

\begin{tabular}{|c|c|c|c|c|c|}
\hline Outcome & Variable & Univariable $P$ value & Risk ratio* & Multivariable $95 \% \mathrm{Cl}$ & $P$ value \\
\hline \multirow[t]{6}{*}{ Death } & Prior aortopulmonary shunt & .17 & & & .72 \\
\hline & Postoperative PA occlusion & .16 & 10.8 & $2.2-53.4$ & .014 \\
\hline & Postoperative main PA pressure & .08 & & & .36 \\
\hline & Postoperative RV/LV pressure ratio & .2 & & & .96 \\
\hline & Primary repair technique & .02 & 39.8 & $5.6-81.2$ & .006 \\
\hline & Reintervention & .18 & & & .48 \\
\hline \multirow[t]{6}{*}{ Reintervention } & Age at operation (y) & .2 & & & .37 \\
\hline & Sex & .11 & & & .48 \\
\hline & Body surface area & .08 & & & .18 \\
\hline & Preoperative right PA Z-score & .07 & & & .09 \\
\hline & Primary repair technique & .13 & & & .16 \\
\hline & Year of operation & .06 & & & .13 \\
\hline \multirow[t]{4}{*}{ PA occlusion } & Age at operation & .04 & & & .64 \\
\hline & Body surface area & .01 & .1 & $0.03-0.54$ & .02 \\
\hline & Prior aortopulmonary shunt & .05 & & & .53 \\
\hline & Aortopulmonary collaterals & .04 & 4.0 & $1.9-6.5$ & .03 \\
\hline
\end{tabular}

$C I$, Confidence interval; $P A$, pulmonary artery; $R V$, right ventricle.

*Risk ratio represents the odds ratio derived from multiple logistic regression analysis for the outcome "PA occlusion," and the hazards ratio obtained from the Cox proportional hazards model for the outcomes "death" and "reintervention." Only variables with $P \leq .2$ in the univariable analysis are shown. All variables tested are listed in the appendix.

of 20 patients $(56 \%)$ had a Fontan procedure completed at last follow-up, and 7 patients had a recent bidirectional Glenn procedure and are awaiting completion of the Fontan circulation. Five patients underwent a bidirectional Glenn shunt but needed subsequent implantation of a modified Blalock-Taussig shunt for recurrent branch PA occlusion, in 2 cases a Fontan attempt failed and was taken down to a bidirectional Glenn shunt, 1 patient died before the Fontan operation, and 1 patient was unavailable for follow-up after PA reconstruction and shunt implantation.

Survival. One patient (3\%) died early and one patient (3\%) died late after repair of PA discontinuity in the setting of a functionally univentricular heart (Table 1). KaplanMeier estimated survivals were $97 \%$ (95\% CI 93-100) at 1 year and 93\% (95\% CI 85-100) at 5 years (Figure 2).

Reinterventions. After construction of PA continuity, 7 patients (19\%) underwent patch enlargement of central PA stenoses during follow-up, and 16 patients (44\%) underwent as many as four interventional balloon dilatations of PA stenoses, in 6 cases including placement of intravascular stents. When surgical and catheter-based procedures were combined, the freedoms from pulmonary arterioplasty after construction of PA continuity were $51 \%$ (95\% CI $38 \%-64 \%)$ at 1 year and $46 \%$ (95\% CI 29\%-63\%) at 5 years (Figure 3). Other reoperations among patients with univentricular anatomy included implantation or revision of a central or modified Blalock-Taussig shunt in 4 cases (11\%), construction of a bidirectional Glenn shunt in 9 cases (25\%), and a modified Fontan operation in 14 cases (39\%).
Kaplan-Meier estimated freedoms from any kind of reoperation were $73 \%(95 \%$ CI $61 \%-85 \%)$ at 1 year and $27 \%$ (95\% CI $11 \%-43 \%$ ) at 5 years. In 16 cases (44\%) APCAs were occluded by intravascular coil placement, and 4 patients had a Fontan baffle fenestration interventionally closed.

Pulmonary circulation. At most recent cardiac catheterization, the median SVC or Fontan baffle pressure was $14 \mathrm{~mm}$ $\mathrm{Hg}$, ranging between 8 and $40 \mathrm{~mm} \mathrm{Hg}$. Median pressures were $14 \mathrm{~mm} \mathrm{Hg}$ (range 7-40 mm $\mathrm{Hg}$ ) in the right PA and 13.5 $\mathrm{mm} \mathrm{Hg}$ (range $4-40 \mathrm{~mm} \mathrm{Hg}$ ) in the left PA. Three patients $(8 \%)$ had a significant $(>5 \mathrm{~mm} \mathrm{Hg}$ ) pressure gradient between a distal branch PA and the SVC or Fontan conduit. The median pulmonary vascular resistance was 2.3 Wood units (range 0.7-11.6 Wood units), and 15 patients (42\%) had a net right-to-left shunt. The mean peripheral oxygen saturation in room air at last follow-up was $86 \% \pm 8 \%$.

Right PA $z$ scores at last follow-up ranged between -4.1 and 1.2 and were lower than -2 in 1 case (3\%). Left PA $z$ scores ranged between -4.4 and 2.1 and were lower than -2 in 4 cases $(11 \%)$. In 16 cases lung perfusion scans had been performed during recent follow-up, and 10 of those scans showed a lung perfusion mismatch exceeding 75:25. In 4 cases one branch PA was found occluded during follow-up and was successfully recanalized, by balloon dilatation and stent placement in 1 patient and surgically in the other 3 .

Risk factor analysis. Results of the univariable analysis indicated that a direct PA-PA anastomosis for construction of PA continuity was associated with better survival $(P=.02$; 
Table 2). Multivariable analysis with the Cox model confirmed that the implantation in a RV-PA homograft was associated with a risk of death approximately 40 times greater than direct PA-PA anastomosis (hazard ratio 39.8, 95\% CI 5.6-81.2, $P=.006)$. In addition, the risk of death was estimated to be more than 10 times higher (hazard ratio 10.8, 95\% CI 2.2-53.4, $P=.014$ ) for patients with postoperative PA occlusion. None of the other variables tested proved to be independent risk factors for death. On the basis of the univariable results, several variables were considered as candidates in the multivariable Cox model for reintervention or reoperation on the PAs, including age at operation, gender, body surface area, preoperative right PA $z$ score, primary repair technique, and year of operation. None of these variables was found to be significantly predictive of PA angioplasty during follow-up. With respect to PA occlusion as an outcome variable, logistic regression revealed four variables (younger age at operation, smaller body surface area, previous aortopulmonary shunt, and the presence of APCAs) that were significant predictors in the univariable analysis. Multiple stepwise logistic regression indicated that a small body surface area (odds ratio $0.1,95 \%$ CI $0.03-0.54, P=.02$ ) and the presence of APCAs (odds ratio 4.0, 95\% CI 1.9-6.5, $P=.03$ ) were significant independent risk factors for PA occlusion.

\section{Discussion}

The main finding of our study is that complex malformations associated with discontinuity of the central PAs can be repaired or palliated with good long-term survival, even in the setting of a Fontan circulation. However, frequent reinterventions are necessary to treat residual or recurrent PA stenoses or hypoplasia. Primary establishment of antegrade PA flow without previous shunt operations results in a favorable outcome and appears to facilitate PA growth better than does initial palliation with systemic-to-PA shunts. Whenever possible, a direct PA-PA anastomosis is preferable, and all collateral vessels should be occluded before or at the time of reconstruction of PA continuity.

\section{Biventricular Repair}

The therapeutic goal for patients with discontinuous PAs is the establishment of an RV-dependent pulmonary circulation with a low postoperative RV-left ventricle pressure ratio and no residual left-to-right shunt. In patients with unilateral discontinuity of otherwise normally sized PAs and a patent right ventricular outflow tract, this can be achieved by connecting the discontinuous PA to the main PA. In these cases it is important to remove any of the ductal tissue that is often present at the origin of the PA when it arises from the aortic arch or innominate or subclavian artery. However, congenitally discontinuous PAs are often hypoplastic. ${ }^{3}$ They frequently show abnormal arborization, with the number of lung segments supplied by branches of the true PAs varying between 1 and $10 .{ }^{1}$ Lung seg- ments not supplied by true PAs are usually supplied by APCAs. APCAs can be incorporated in the pulmonary circulation by surgical unifocalization procedures but as primary muscular arteries remain structurally different from true PAs. We therefore chose to exclude patients in whom pulmonary blood flow was partially dependent on multiple APCAs, and focused on patients who had discontinuous PAs with normal distal arborization. Patients who will eventually undergo a biventricular repair are believed to benefit from early reconstruction of PA continuity, reducing the severity of right ventricular pressure load after separation of pulmonary and systemic circulations. Since the introduction of balloon dilatation for stenotic or hypoplastic PAs and transcatheter coil interruption of APCAs, a staged approach to the management of patients with pulmonary atresia and diminutive or discontinuous PAs has evolved at our institution. It consists of early surgical relief of right ventricular outflow tract obstruction, leaving the VSD open or inserting a fenestrated VSD patch that allows for right-to-left shunting, followed by interventional catheterization to dilate stenotic peripheral PAs and occlude redundant APCAs. ${ }^{10}$ The VSD is then closed when pulmonary vascular resistance has reached an acceptable level. ${ }^{11}$ For patients with discontinuous PAs, however, initial shunt palliation is sometimes unavoidable. In such cases a 3.5- or 4-mm polytetrafluoroethylene graft is interposed between the subclavian artery and the stump of the ipsilateral PA, and this procedure is repeated on the opposite side if necessary. The objective is to stimulate growth of these PAs, eventually connect the left and right PAs, and finally connect the reconstituted PAs to the RV with a valved homograft. After enlargement of both left and right intraparenchymal pulmonary branches is documented angiographically (usually after 6 to 12 months), a conduit is interposed between the right and left hilar PAs, and a valved aortic homograft is inserted that connects the RV with the nonvalved conduit. Not surprisingly, we found that a direct PA-PA anastomosis is associated with better outcome than is interposition of a tube graft, but a tension-free anastomosis is not always possible and often requires extensive mobilization of both PAs. The presence of APCAs proved to be a risk factor for PA occlusion. This finding supports the hypothesis that competing flow from collateral vessels promotes stasis in the native PAs, increasing the risk of thrombosis and hence occlusion. It underscores the need for early closure of all redundant APCAs by either surgical ligation or coil placement. The most interesting finding is perhaps the evidence that patients without previous shunt implantation have better PA development, as indicated by PA $z$ scores and right ventricular pressure load, than do patients who have undergone initial shunt palliation. Implantation of an aortopulmonary shunt in a hypoplastic PA facilitates growth by increasing both flow and pressure. On the other hand, the effects of a chronic left-to-right shunt with nonpulsatile flow on long-term pulmonary vascular development remain largely unclear. We believe that early establish- 
ment of a pulmonary blood supply that is as physiologically normal as possible is the best strategy to facilitate PA growth and avoid the development of pulmonary vascular disease. This approach has also led to favorable results in the treatment of other congenital malformations with hypoplastic true PAs and multifocal pulmonary blood supply. ${ }^{12-14}$ Even when a VSD cannot be closed at the time of establishment of PA continuity and the central PAs are therefore perfused with mixed blood at elevated pressure, the antegrade pulsatile flow pattern may still have beneficial effects on PA development as compared with shunt perfusion.

One subset of patients has increased blood flow in the discontinuous PAs, as seen in cases of isolated, unobstructed origin of one PA from the ascending aorta or truncus arteriosus variants. ${ }^{15,16}$ Early repair is mandatory to avoid the development of pulmonary vascular disease and heart failure and can usually be achieved by direct anastomosis of the isolated discontinuous PA to the main PA or contralateral branch PA. ${ }^{17,18}$ As has been pointed out by Mee, ${ }^{19}$ however, a two-stage approach is often necessary when the right PA arises from the ascending aorta and the left PA arises from the descending aorta or when one lung is supplied by multiple APCAs requiring unifocalization, but the latter cases were excluded from our study.

\section{Single Ventricle or Fontan Repair}

For patients with complex congenital malformations who will eventually undergo a Fontan procedure, PA discontinuity poses a particularly challenging problem, because any residual or recurrent obstruction to pulmonary blood flow may lead to failure of the Fontan circulation. There are numerous reports of operative and long-term results after a bidirectional Glenn or Fontan procedure, but there is virtually no information regarding the feasibility of a Fontan circulation after reconstruction of discontinuous PAs. ${ }^{20}$ However, it has become clear that even a moderate degree of PA distortion, as is often seen after implantation of systemic-to-PA shunts, is a risk factor for failure of a modified Fontan operation. ${ }^{21}$ Our cohort included a number of patients in whom PA discontinuity developed after implantation of central aortopulmonary shunts, and it is our policy to avoid central shunts in favor of modified Blalock-Taussig shunts. In cases of congenital PA discontinuity, early restoration of PA continuity is deemed mandatory to induce sufficient PA growth and thus lower pulmonary vascular resistance. When acquired PA discontinuity develops after implantation of a systemic-to-PA shunt in patients with univentricular physiology, early restoration of PA continuity with generous patch enlargement of the central PAs is recommended. We were surprised to find that survival of patients with single ventricle and PA discontinuity was quite favorable; however, frequent reinterventions were necessary to recanalize thrombosed PAs or dilate residual or recurrent stenoses. Because even mild PA stenoses in patients with a cavopulmonary anastomosis compromise PA blood flow to a much greater extent than in patients with pulsatile pulmonary blood flow, the incidence of a significant perfusion mismatch was higher than among patients with biventricular physiology. However, PA perfusion scans in the setting of a Fontan circulation can show a perfusion mismatch despite completely unobstructed PA anatomy, depending on the site of tracer injection and the distribution of inferior and superior caval blood flows, so the incidence of a true perfusion mismatch is probably overestimated. We conclude that a Fontan circulation can be established with good operative and medium-term results in patients with congenital or acquired PA discontinuity.

\section{Limitations of the Study}

One limitation of our study is the heterogeneity of underlying intracardiac defects and variation in the severity of the PA malformation. The spectrum of congenital cases of PA discontinuity extends from isolated unilateral discontinuity of well-developed PAs to atretic right ventricular outflow tract with absence of the main PA; it also includes a small number of cases of increased pulmonary blood flow (hemitruncus arteriosus). Caution should be exercised when interpreting the comparison between primary repair and previous shunt palliation, because it can be argued that mainly patients with severe forms of PA discontinuity underwent previous shunt implantation and only those with more favorable anatomy underwent primary repair. It should also be remembered that this study includes only patients with discontinuous PAs and normal peripheral arborization, so the conclusions cannot be readily extrapolated to patients with continuous but hypoplastic PAs or with an APCA-dependent pulmonary blood supply.

\section{References}

1. Shimazaki Y, Maehara T, Blackstone EH, Kirklin JW, Bargeron LM. The structure of the pulmonary circulation in tetralogy of Fallot with pulmonary atresia. J Thorac Cardiovasc Surg. 1988;95:1048-58.

2. Robin E, Silberberg B, Ganguly SN, Magnisalis K. Aortic orgin of the left pulmonary artery: variant of tetralogy of Fallot. Am J Cardiol. 1975;35:324-9.

3. Rabinovitch M, Herrera-deLeon V, Castaneda AR, Reid L. Growth and development of the pulmonary vascular bed in patients with tetralogy of Fallot with or without pulmonary atresia. Circulation. 1981;64:1234-49.

4. Collet RW, Edwards JE. Persistent truncus arteriosus. A classification according to anatomic types. Surg Clin North Am. 1949;29:1245-70.

5. Van Praagh R, Van Praagh S. The anatomy of common aorticopulmonary trunk (truncus arteriosus communis) and its embryologic implications. Am J Cardiol. 1965;16:406-25.

6. Keane JF, Maltz D, Bernhardt WF, Corwin RD, Nadas AS. Anomalous origin of one pulmonary artery from the ascending aorta: diagnostic, physiological and surgical considerations. Circulation. 1974;50:588-94.

7. Penkoske PA, Castaneda AR, Fyler DC, Van Praagh R. Origin of pul- 
monary artery branch from ascending aorta. Primary surgical repair in infancy. J Thorac Cardiovasc Surg.1983;85:537-45.

8. Kutsche LM, Van Mierop LH. Anomalous origin of a pulmonary artery from the ascending aorta: associated anomalies and pathogenesis. Am J Cardiol. 1988;61:850-6.

9. Waldman JD, Karp RB, MD, Gittenberger-de Groot AC, Agarwala B, Glagov S. Spontaneous acquisition of discontinuous pulmonary arteries. Ann Thorac Surg. 1996;62:161-8.

10. Rome JJ, Mayer JE, Castaneda AR, Lock JE. Tetralogy of Fallot with pulmonary atresia: rehabilitation of diminutive pulmonary arteries. Circulation. 1993;88:1691-8.

11. Reddy VM, Petrossian E, McElhinney DB, Moore P, Teitel DF, Hanley FL. One-stage complete unifocalization in infants: when should the ventricular septal defect be closed? J Thorac Cardiovasc Surg. 1997;113:858-68.

12. Tchervenkov CI, Salasidis G, Cecere R, Beland MJ, Jutras L, Pacquet $\mathrm{M}$, et al. One-stage midline unifocalization and complete repair in infancy versus multiple-stage unifocalization followed by repair for complex heart disease with aortopulmonary collaterals. $J$ Thorac Cardiovasc Surg. 1997;114:727-37.

13. McElhinney DB, Reddy VM, Hanley FL. Tetralogy of Fallot with major aortopulmonary collaterals: early total repair. Pediatr Cardiol. 1998;19:289-96.

14. Reddy VM, McElhinney DB, Amin Z, Moore P, Parry AJ, Teitel DF, et al. Early and intermediate outcome after repair of pulmonary atresia with ventricular septal defect and major aortopulmonary collateral arteries: experience with 85 patients. Circulation. 2000;101:1826-32.

15. Van der Horst RL, Gotsman MS. Type 3c truncus arteriosus: case report with clinical and surgical implications. Br Heart J. 1974;36:1046-8.

16. Guadalupi P, Spadoni I, Vanini V. Repair of hemitruncus with autologous arterial ring and valved bioconduit. Ann Thorac Surg. 2000, 70:1708-10.

17. Mair DD, Ritter DG, Danielson GK, Wallace RB, McGoon DC. Truncus arteriosus with unilateral absence of a pulmonary artery: criteria for operability and surgical results. Circulation. 1977;55:641-7.

18. Soylu M, Demir AD, Tikiz H, Kisacik H, Korkmaz S. Left hemitruncus associated with tetralogy of Fallot: a case report. Catheter Cardiovasc Interv. 2000;51:58-60.

19. Mee RB. Surgical repair of hemitruncus: principles and techniques. $J$ Card Surg. 1987;2:247-56.

20. Stamm C, Friehs I, Mayer JE Jr, Zurakowski D, Triedman JK, Moran AM, et al. Long-term results of the lateral tunnel Fontan operation. $J$ Thorac Cardiovasc Surg. 2001;121:28-41.

21. Gentles TL, Mayer JE Jr, Gauvreau K, Newburger JW, Lock JE, Kupferschmid JP, et al. Fontan operation in five hundred consecutive patients: factors influencing early and late outcome. $J$ Thorac Cardiovasc Surg. 1997;114:376-91.

\section{Discussion}

Dr Vaughn A. Starnes (Los Angeles, Calif). Stamm and colleagues have presented a retrospective review of 102 patients undergoing PA reconstruction for discontinuous intrapericardial PAs. The methods of reconstruction included direct PA-PA anastomosis in 33 cases, tube graft interposition, either polytetrafluoroethylene or homograft, in 47 cases, and PA implantation into either the main PA or the conduit used for reconstruction in 22 cases. Cardiac defects were mainly tetralogy of Fallot with either stenosis or atresia, with 66 patients undergoing biventricular repair and another 33 patients undergoing single-ventricle reconstruction. PA anatomy consisted of ductal-dependent flow or collateral flow in $80 \%$ of cases, whereas only $20 \%$ of patients had main PA flow.

The main findings of this study were that complex malformations with discontinuous PAs can be successfully repaired provided the expectation of reintervention is accepted. This is slightly different than presented, because in reading the article I added up surgical reintervention and balloon reintervention and determined that only $11 \%$ of patients were free of reintervention at the end of 5 years. In point of fact, $67 \%$ of them had as many as 5 balloon dilatations, which leads me to my first question.

Dr Stamm, was there a type of repair or reconstruction or material use that biased the patient toward more reintervention?

Dr Stamm. We did examine the type of repair, either tube graft interposition, direct anastomosis, or implantation into a right ventricular homograft, but we did not find any significant influence of any of those techniques on the incidence of reintervention in the multivariable analysis.

Dr Starnes. Although the initial supposition was that the establishment of early antegrade flow and the avoidance of a shunt would allow better growth and fewer interventions, this was not proved by your data. In light of this information, how would you handle the 4-kg neonate with discontinuous 4-mm intrapericardial PAs at your institution today? Would the operation be a PA-PA direct anastomosis and shunt, or would it be a PA-PA anastomosis with homograft reconstruction of the outflow tract? Why?

Dr Stamm. Because the hemodynamics allow a primary repair in the situation that you just described, we would go for primary implantation of a right ventricular tube PA homograft together with end-to-end anastomosis of both PAs if possible, or direct implantation of the PAs into the homograft if not, leaving the VSD open if necessary. This way we would open the way for the interventional cardiologist to dilate those PAs and decrease pulmonary vascular resistance, so that we can close the VSD later.

Dr Starnes. One of your risk factors that dropped out with the multivariate analysis was PA occlusion onto a graft or homograft reconstruction. I'm really asking this question in light of that factor alone, because sometimes we are biased by our own beliefs that antegrade flow is the better way to reconstruct the circulation but I'm not sure our data today prove that. You are to be congratulated in that you tried to take a difficult group of patients and boil it down into a simple subset, if you would, of intrapericardial discontinuous PAs.

Dr Francisco J. Puga (Rochester, Minn). I also congratulate you for an excellent presentation on an important subject. The key issue in reestablishing confluence and gauging the success of such an operation is distribution of pulmonary blood flow; that is, if you don't really correct much in terms of the distribution of pulmonary blood flow, you haven't really accomplished much. Right to left ventricular pressure ratio or initial parameters of right ventricular function are probably not too significant if one PA is normal in size and the other one is extremely small, because the capacitance of a single lung, at least at those ages, is going to be adequate.

Our experience with reconstructive confluence of a highly hypoplastic PA to normal size PAs is a small vessel will not growit may remain patent-and it's not going to create problems until much later in life. We see adult patients coming to our adult cardiac clinics who have had hypertension develop gradually on the right side and have an underdeveloped PA still patent but connected to the central PAs. Because of this our attack has been to prepare the PA, if it's small, by preliminary shunting in an effort to induce growth. Only when growth is more or less equivalent to that of the other PAs do we then proceed with reconstruction of the pulmonary confluence. We think that the test of distribution of pulmonary blood flow is critical, because otherwise I don't think we are accomplishing much except to say that we have restored the confluence. 
Have you noticed the same phenomenon of lack of growth of these small PAs? If so, how do you intend to deal with that problem?

Dr Stamm. Thank you for your comments and your questions. We did actually observe the same phenomenon. I am aware of at least 3 patients who did initially have a marked mismatch in size of the discontinuous PAs. Even after the establishment of confluence, now, as long as 15 years after the initial operation, the initially hypoplastic PA is still small, and flow to the respective lung is significantly impaired.

With respect to our analysis of whether a primary repair or a shunt is the better option, I have to admit that the major limitation is that patients with a less favorable anatomy to begin with tended to get shunts because there was no other option. Whereas the patients who had primary correction, of course, tended to be those with more favorable anatomy. I think that this is a significant limitation of our analysis and certainly weakens the conclusion.

Dr John J. Lamberti (New York, NY). I have a comment and a question. In this group of patients, particularly infants born with single ventricle, you may find PAs that arise from a ductus that look as though they can be put directly together. When you operate, however, there is ductal tissue extending out to the hilum. Then you have to use some kind of material, either pericardium or synthetic material, to create a confluence. One of the problems that we've seen is that when we perform follow-up angiography in these patients, the PA is narrow in a location that we had thought was free of ductal tissue. If you've sewn it to a piece of polytetrafluoroethylene, you're pretty confident about its size when you completed the operation.

My own bias in most of these cases is to have an early catheterization, as soon as your interventionalist and you are comfortable with dilating or stenting. If I do this type of operation in any patient, but especially in the patient with a single ventricle, I want a restudy in 6 to 8 weeks. The corollary of this approach is that in the singleventricle group you want to get to the bidirectional cavopulmonary shunt as early as possible, so that the interventional cardiologist can have access to the PAs. Do you have a recommendation for timing of angiography, and did you have a protocol for that with these patients?

Dr Stamm. We do have a protocol that we developed over the last decade or so at Children's Hospital. We try to catheterize those patients within 3 to 6 months after establishment of PA continuity and, if necessary, perform balloon dilatations and prepare the patients for separation of pulmonary and systemic circulations.

Dr Lamberti. I think that the incidence of occluded PAs would be diminished by early restudy. If the 1-year follow-up study reveals that the PA is occluded, and presumably it was patent when you left the operating room, an earlier restudy might have salvaged the artery.

\section{Appendix}

Variables tested as possible predictors of death, reintervention, and PA occlusion

Age at operation (continuous)

Sex

Body surface area (continuous)

Prior systemic-to-pulmonary artery shunt

Primary establishment of antegrade PA flow

Diagnosis

Tetralogy of Fallot with pulmonary atresia

Tetralogy of Fallot with patent right ventricular outflow tract

Other forms of pulmonary atresia

Tricuspid atresia

Truncus arteriosus

Others

Univentricular anatomy

Biventricular anatomy

Preoperative RV/LV pressure ratio (continuous)

Preoperative right PA Z-score (continuous)

Preoperative left PA Z-score (continuous)

Presence of aortopulmonary collaterals

Surgical repair technique

Direct PA-PA anastomosis

Tube graft interposition

Implantation in RV-PA homograft

Year of operation (continuous)

Postoperative lung perfusion mismatch $>75 \% / 25 \%$

Postoperative RV/LV pressure ratio (continuous)

Postoperative main PA pressure (continuous)

Postoperative PA occlusion

Reintervention 